\section{$\$$ Research Square}

\title{
Active control of micrometer plasmon propagation in suspended graphene
}

\section{Qing Dai ( $\nabla$ daiq@nanoctr.cn )}

National Center for Nanoscience and Technology https://orcid.org/0000-0002-1750-0867

Hai Hu

National Center for Nanosicence and technology https://orcid.org/0000-0002-4243-2333

\section{Renwen Yu}

ICFO-Institut de Ciencies Fotoniques

Debo Hu

National Center for Nanoscience and Technology https://orcid.org/0000-0001-9432-1670

Alexander McLeod

Columbia University https://orcid.org/0000-0002-5232-634X

Pablo Alonso-González

University of Oviedo https://orcid.org/0000-0002-4597-9326

\section{Xiangdong Guo}

National Center for Nanoscience and Technology

Hanchao Teng

National Center for Nanoscience and Technology

Chan Li

National Center for Nanoscience and Technology

$\mathrm{Na}$ Chen

National Center for Nanoscience and Technology

Xinzhong Chen

Stony Brook University

\section{Ziheng Yao}

Stony Brook University https://orcid.org/0000-0002-8296-1272

\section{Yunpeng Qu}

National Center for Nanoscience and Technology

\section{Zhongjun Li}

National Center for Nanoscience and Technology, China

\section{Jianing Chen}

institute of physics, chinese academy of science

\section{Zhipei Sun}

Aalto University https://orcid.org/0000-0002-9771-5293

Mengkun Liu 
Stony Brook University

Javier Garcia de Abajo

ICFO

Xiaoxia Yang

National center for nanoscience and technology https://orcid.org/0000-0002-0670-053X

Letter

Keywords: graphene, photonic devices, plasmons

Posted Date: April 28th, 2021

DOl: https://doi.org/10.21203/rs.3.rs-448924/v1

License: (c) (1) This work is licensed under a Creative Commons Attribution 4.0 International License. Read Full License 


\section{Active control of micrometer plasmon propagation in suspended graphene}

Hai Hu, ${ }^{1,2 *}$ Renwen Yu, ${ }^{3}$ Debo Hu, ${ }^{1,2}$ Xiaoxia Yang, ${ }^{1,2}$ A. S. McLeod, ${ }^{6}$ Pablo AlonsoGonzález, ${ }^{7}$ Xiangdong Guo, ${ }^{1,2}$ Hanchao Teng, ${ }^{1,2}$ Chi Li, ${ }^{1,2} \mathrm{Na}$ Chen, ${ }^{1,2}$ Xinzhong Chen, ${ }^{5}$ Ziheng Yao, ${ }^{5}$ Yunpeng Qu, ${ }^{1,2}$ Zhenjun Li, ${ }^{1,2}$ Jianing Chen, ${ }^{8}$ Zhipei Sun, ${ }^{9,}{ }^{10}$ Mengkun Liu, ${ }^{5}$ F. Javier García de Abajo, ${ }^{3,4}$ Qing Dai ${ }^{1,2 *}$

1 CAS Key Laboratory of Nanophotonic Materials and Devices, CAS Key Laboratory of Standardization and Measurement for Nanotechnology, CAS Center for Excellence in Nanoscience, National Center for Nanoscience and Technology, Beijing 100190, P. R. China.

2 University of Chinese Academy of Sciences, Beijing 100049, P. R. China.

3 ICFO-Institut de Ciencies Fotoniques, The Barcelona Institute of Science and Technology, 08860 Castelldefels (Barcelona), Spain.

4 ICREA-Institució Catalana de Recerca i Estudis Avançats, Passeig Lluís Companys 23, 08010 Barcelona, Spain.

5 Department of Physics and Astronomy, Stony Brook University, Stony Brook, New York 11794, USA.

6 Department of Physics, Columbia University, New York, NY, USA.

7 Departamento de Física, Universidad de Oviedo, Oviedo, Spain.

8 The Institute of Physics, Chinese Academy of Sciences, P.O. Box 603, Beijing, China.

9 Department of Electronics and Nanoengineering Aalto University Tietotie 3, FI-02150 Espoo, Finland.

10 QTF Centre of Excellence Department of Applied Physics Aalto University FI-00076 Aalto, Finland.

*e-mail: daiq@nanoctr.cn, javier.garciadeabajo@icfo.eu, huh@ nanoctr.cn 
Doped graphene supports ultrahigh-confined, low-loss, and tunable surface plasmons that hold great potential for developing infrared optoelectronic nanodevices with unprecedented capabilities. However, due to the two-dimensional character of this material, graphene plasmons have been invariably studied in supported samples so far. The substrate provides stability for graphene but often causes undesired interactions (such as dielectric loss, phonon hybridization, and impurity scattering) that compromise the quality of graphene plasmons, and limits intrinsic flexibility of the material. Here, we demonstrate the visualization of plasmons in suspended graphene at room temperature, exhibiting highquality factor $Q \sim 33$ and long propagation length $>3 \mu \mathrm{m}$. Suspended graphene also provides an exceptional undisturbed plasmonic environment to exploit the behavior of the intrinsic flexibility under extrinsic modification. We introduce the graphene suspension height as a novel plasmonic tuning knob that enables in situ change of the dielectric environment and substantially modulates the plasmon wavelength, propagation length, and group velocity. Such active control of micrometer plasmon propagation facilitates near-unity-order modulation of nanoscale energy flow that serves a new plasmonic switch with an on-off ratio above 14. The suspended graphene plasmons possess long propagation length, high tunability, and controllable energy transmission simultaneously, opening up new horizons for their integration in practical photonic devices. 
Graphene plasmons (GPs), the collective oscillations of Dirac-fermion electrons in doped graphene, enable extremely subwavelength light confinement in the infrared (IR) and terahertz frequency domains, ${ }^{1,2,3}$ offering an ideal platform to support high-speed, ${ }^{4}$ low-damping,, 6 actively-tunable energy transport at the nanoscale. ${ }^{7} 8$ Importantly, GP properties can be dynamically tuned by changing the graphene Fermi energy through electrical gating, which finds applications ranging from light modulation to light detection and sensing. ${ }^{9}$ 10, 11 Additionally, extensive efforts have been devoted to creating passive graphene components for plasmonic circuitry, such as waveguides, resonators, and couplers. ${ }^{12}$ However, the current state-of-the-art of GPs is far from the real-world applications due to their short propagation length, limited tunability, and lack of energy flow controllability. This is because the performance of GPs is limited by a supporting substrate underneath graphene, which brings about extrinsic damping pathways of GPs, including dielectric loss, phonon hybridization, and impurity scattering. Such extrinsic damping comes mainly from the natural IR phonons that reside in the chosen substrates, such as $\mathrm{SiO}_{2}$ and BN. ${ }^{5,6}$ Even at cryogenic temperatures, dielectric losses from the environment still contribute a significant damping that cannot be overcome by improving the intrinsic properties of graphene. ${ }^{13}$ The dielectric permittivity and phonon hybridization also restrict the operating frequency bandwidth of GPs. Besides, when plasmons propagate across different substrates, electromagnetic energy is affected by the dramatic change of the local dielectric environment, thereby causing reflection, transmission, and radiative out-coupling associated with the plasmonic impedance mismatch between the two different dielectric environments. ${ }^{14,15,16,17}$ Therefore, the design of a dielectric environment to address the aforementioned challenges in one system is essential to bring GPs close to real applications.

Suspended graphene with very high carrier mobility approaching ballistic transport and long carrier relaxation time ${ }^{18,19}$ serves as an ideal platform to avoid dielectric losses from the substrate. Notably, suspended graphene structures have been widely explored in photodetectors, ${ }^{6}$ ultrafast photocurrents and terahertz generation, ${ }^{20}$ visible light emission, ${ }^{21}$ nanomechanical resonators, ${ }^{22}$ and thermal transport devices. ${ }^{23}$ Additionally, without the presence of a high-index dielectric substrate, the plasmon wavelength can be effectively pushed to the micron scale, achieving longerdistance propagation for a given damping rate. ${ }^{2}{ }^{3}$ Here, we demonstrate that remarkable improvement in the quality factor and propagation length of GPs can be achieved in suitably designed suspended graphene plasmonic structures. Furthermore, a broad tunability of the plasmon 
dispersion, propagation length, phase and group velocities, and energy flow is demonstrated by merely in situ adjusting the suspension height, leading to a change of dielectric environment that is difficult to achieve in substrate-supported plasmonic structures. Based on the active control of micrometer-propagation-plasmon, we have implemented a novel switch for near-unity-order control of plasmonic energy.

a

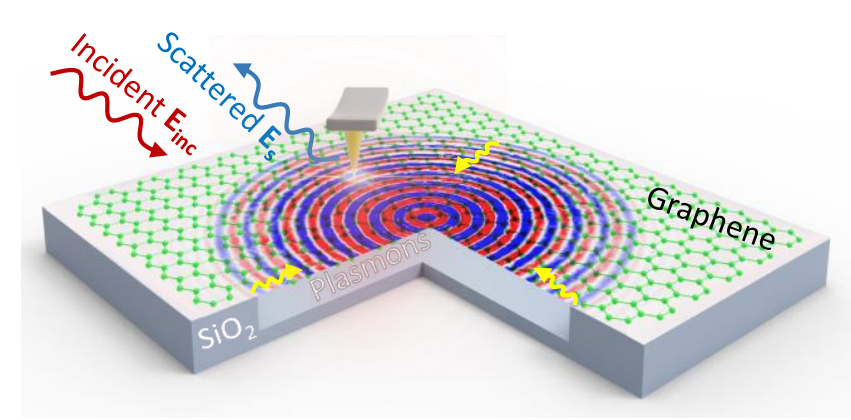

C

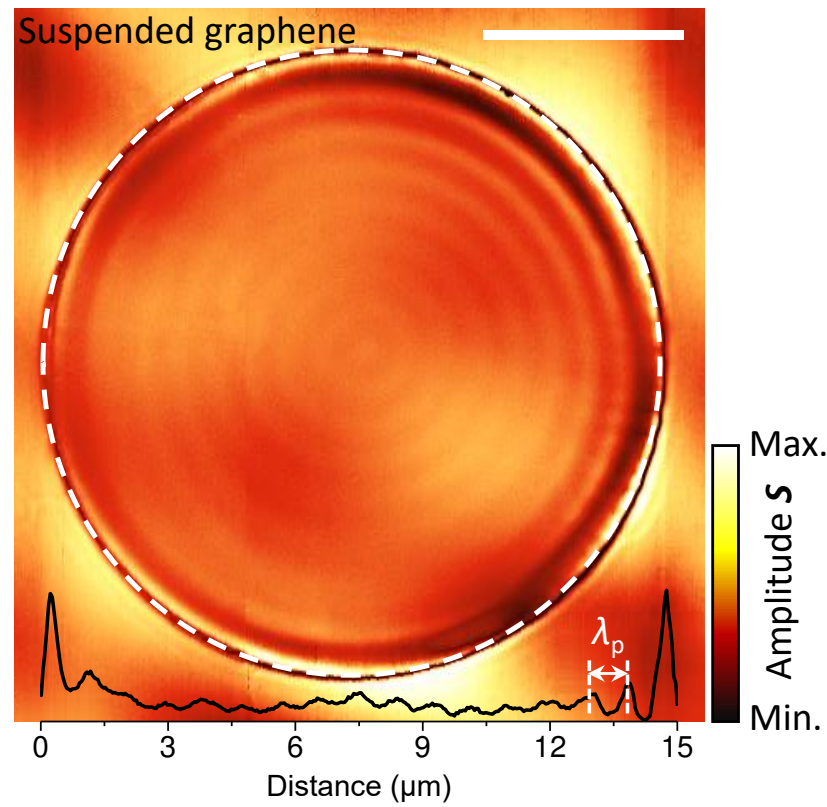

b

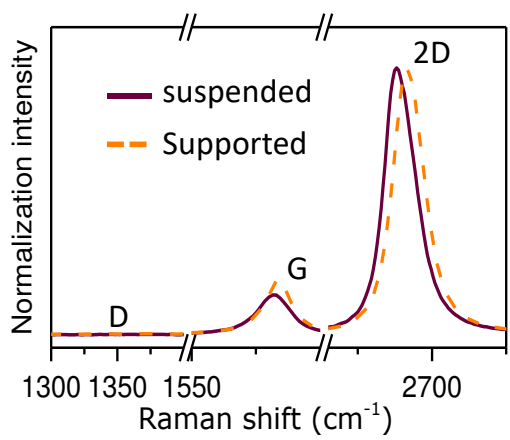

d

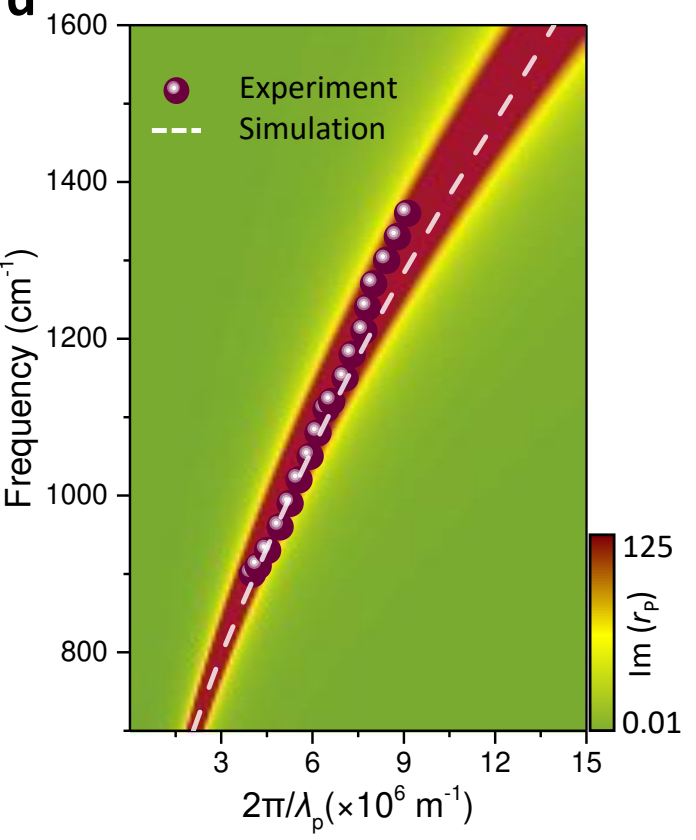

Figure 1. Plasmonic response and intrinsic dispersion in suspended graphene structures. (a) Sketch of experimental arrangement for launching and detecting plasmons propagating in suspended graphene. The tip and sample are illuminated with a focused infrared light (with wavelength $\lambda_{0}$ and field $E_{\text {inc }}$ ), which can induce dipoles around the hole edge, thus efficiently exciting the graphene plasmons (yellow arrows). The tip subsequently scatters the plasmon, and a distant detector measures the scattered field $\mathrm{E}_{S}$. Note that we use defocus processing to enlarge the spot size to $\sim 30$ microns and enhance the surrounding edge launching efficiency further. (b) Raman spectra of suspended and $\mathrm{SiO}_{2}$-supported regions of high-quality undoped graphene. (c) Near-field optical signal at $\lambda_{0}=10.87 \mu \mathrm{m}\left(920 \mathrm{~cm}^{-1}\right)$ light wavelength from a two-dimensional 
scan across the suspended graphene area with a circular diameter $\Phi=15 \mu \mathrm{m}$ and a suspension height $\sim 450 \mathrm{~nm}$. The interface between suspended and supported graphene regions is marked with a white dashed circle. The black line at the bottom shows the near-field profile along with a horizontal cut through the center. The graphene Fermi energy is $E_{\mathrm{F}} \sim 0.68 \mathrm{eV}$. The scale bar indicates $5 \mu \mathrm{m}$. (d) Experimentally measured (symbols, from another device with $\Phi=2.5 \mu \mathrm{m}$ and $E_{\mathrm{F}} \sim 0.9 \mathrm{eV}$, shown in Figure S4) and simulated (white dashed curve, details in Methods) dispersion of suspended GPs. The loss function $\operatorname{Im}\left\{r_{\mathrm{p}}(\mathrm{q}, \omega)\right\}$ calculated from the reflection coefficient $r_{\mathrm{p}}$ in the random-phase approximation (RPA, details in Methods) is shown as a pseudo-color-plot background.

As schematically shown in Figure 1a, we deposit mechanically exfoliated graphene flakes onto a $\mathrm{SiO}_{2} / \mathrm{Si}$ substrate perforated with circular dimples of different diameters in the $30 \mathrm{~nm}$ to 50 $\mu \mathrm{m}$ range (see also Supplementary Information Figure S1). Our fabrication procedure (details in Methods) avoids contamination by chemical agents, as well as mechanical damage from the surface tension of the liquid to maintain intact the intrinsic properties of graphene during the transfer process. Circular dimples are mainly used to prevent wrinkles and achieve better control of the suspended structure. The holes underneath suspended graphene are filled with $\mathrm{N}_{2}$ gas to avoid the collapse of the carbon monolayer and change its distance to the substrate by controlling the gas pressure. The samples are then sealed in a chamber with $\mathrm{NO}_{2}$ gas (concentrations are varied between $25 \sim 75 \%$ in $\mathrm{N}_{2}$ atmosphere) for several hours to achieve a high doping level by surface adsorption of gas molecules, which act as electron acceptors. ${ }^{24,25}$ The monolayer thickness of the graphene used in this work is confirmed by the Lorentzian profiles of the 2D peak in its Raman spectra (Figure $1 \mathrm{~b}$ and Figure S2), while the absence of a defect-induced D band $\left(1350 \mathrm{~cm}^{-1}\right)$ indicates a high quality of the samples. By comparison, the $\mathrm{G}$ peak of suspended graphene redshifts by $2.1 \mathrm{~cm}^{-1}$, indicating unnoticeable strain (only $0.014 \%$ ) induced in the carbon monolayer by our suspended structures. Besides, the Raman ratio $I_{2 \mathrm{D}} / I_{\mathrm{G}}$ decreases significantly from 5 in suspended graphene to 3 in supported graphene due to the scattering of graphene electrons caused by charged impurities from $\mathrm{SiO}_{2}$ substrate. The doping level can be directly controlled through the gas concentration and doping time (Supplementary Information Figure S2), which can shift the graphene Fermi energy $E_{\mathrm{F}}$ up to $\sim 0.9 \mathrm{eV}$. The resulting Fermi energy is much higher than that provided by the commonly employed electrostatic gating (typically changing the graphene carrier density by up to $\sim 4 \times 10^{12} \mathrm{~cm}^{-2}$, corresponding to a Fermi energy shift of $\sim 0.3 \mathrm{eV}$ from the neutrality point). We find that plasmons remain almost unchanged for several hours and gradually degrade after a few days due to the desorption of $\mathrm{NO}_{2}$ gas molecules. Interestingly, the plasmonic effect 
can be effectively turned off by thermal enhancement of desorption in our experiments (e.g., heating the sample at $100{ }^{\circ} \mathrm{C}$ for $5 \min$ in $\mathrm{N}_{2}$ atmosphere).

We use a scattering-type scanning near-field optical microscope (s-SNOM) with a tunable quantum cascade laser operating in the $890-2500 \mathrm{~cm}^{-1}$ range to image propagating plasmons in graphene. The infrared (IR) light beam is focused on a metallic atomic force microscope (AFM) cantilever probe tip (Figure 1a). The backscattered near-field optical signal can be recorded simultaneously with the topography. The broad scan range of s-SNOM (up to $50 \times 50 \mu \mathrm{m}^{2}$ ) is critical for the characterization of long-distance propagation. In previous studies, it was well established that plasmon polaritons manifest as a periodic modulation (fringes) of the observed near-field signal as a function of the tip position relative to graphene edges and other surface features. $^{2,} 3$ Furthermore, s-SNOM can distinguish tip- and edge-launched plasmons, ${ }^{9}$ thus providing a valuable platform for characterizing plasmon propagation, reflection, and transmission. ${ }^{14,15}$

We first explore plasmon polaritons propagating on a large-area suspended graphene sample $\left(E_{\mathrm{F}}=\sim 0.68 \mathrm{eV}\right)$ with an IR laser operating at $\lambda_{0}=10.87 \mu \mathrm{m}\left(920 \mathrm{~cm}^{-1}\right)$. In the near-field nanoscopy image shown in Figure 1c, plasmonic fringes remarkably cover the entire large suspended graphene area (with a circular diameter $\Phi=15 \mu \mathrm{m}$ ). The corresponding optical and topography micrographs are shown in Figure S1. The wavelength $\lambda_{\mathrm{p}}$ of the suspended GP is calculated to be $938 \mathrm{~nm}$ from analytical RPA theory ${ }^{26}$ and numerical simulations ${ }^{27}$, a value that agrees well with the experimentally observed distance between two adjacent fringes in Figure 1c. As a salient feature of suspended GPs, we remark that their plasmon wavelength is much longer than that of graphene samples supported by substrates. ${ }^{2,3,5}$ Besides, inspection of Figure 1c reveals that the plasmonic oscillations extend $7.5 \mu \mathrm{m}$ beyond the edge of the dimple; propagation length is limited by the sample size and far exceeding previous records on any other substrates at room temperature. $^{2,3,5}$ In contrast, the nanoscopy images of suspended graphene without doping are featureless (Supplementary Information Figure S3).

To investigate the plasmon dispersion as a function of the wave vector $q_{1}$ and photon energy $\omega$, a smaller suspended structure (diameter $\Phi=2.5 \mu \mathrm{m}$ ) is chosen to obtain more accurately measured near-field amplitude images. Fringe patterns can be observed with a periodicity corresponding to $\lambda_{\mathrm{p}} / 2$, which is confirmed by our electromagnetic simulations (Supplementary 
Information Figure S4 and S5). The plasmon dispersion relation extracted from experimental measurements (dots in Figure 1d) is plotted and compared with RPA calculations (pseudo-colorplot background). ${ }^{26}$ Fringes of two distinct periodicities $\left(\lambda_{\mathrm{p}}\right.$ and $\left.\lambda_{\mathrm{p}} / 2\right)$ appear in Figure $1 \mathrm{c}$ and $\mathrm{S} 4$, respectively. The $\lambda_{\mathrm{p}} / 2$-period fringes can be assigned to tip-launched plasmons reflected at the edge of the suspended area and subsequently out-coupled to radiation by the tip. ${ }^{2}$ The plasmon edge-reflection is caused by the mismatch of capacitive coupling across dielectric interfaces, mainly determined by the difference in the wave vectors of plasmons between substrate-supported and suspended regions (more details in the discussion of Figure 4 below). The $\lambda_{\mathrm{p}}$-period fringes can also be produced by edge-launched plasmons propagating to the tip and being out-coupled ${ }^{9}$. We note that the efficiency of plasmon excitation at the edges depends on the combined effect of dielectric polarization ${ }^{28}$ and the size and shape of the hole edge (Supplementary Information Note 1). ${ }^{29}$

With the ultrahigh doping levels enabled by suspended graphene, we can access a wide range of plasmon wavelengths, ranging from $850 \mathrm{~nm}$ to $1560 \mathrm{~nm}$ with different excitation frequencies in our suspended graphene sample. Moreover, the plasmon dispersion relation shown in Figure 1d presents a relatively steep slope, eventually covering excitations by light frequencies up to 1400 $\mathrm{cm}^{-1}$. The operational frequencies are limited by Fermi energies and are generally restricted up to $1000 \mathrm{~cm}^{-1}$ by electrical gating. Besides, suspended graphene can be easily tailored into different shapes and sizes of nanoresonators by engineering the shape of the hole carved in the substrate rather than patterning graphene itself, thus offering a unique opportunity to manipulate plasmonic propagation and localization. For example, the hole size in the substrate can be reduced down to $<50 \mathrm{~nm}$, providing robust on-demand hotspots that are appealing for numerous applications in the field of nanophotonics (Supplemental materials Figure S6). ${ }^{12}$ 

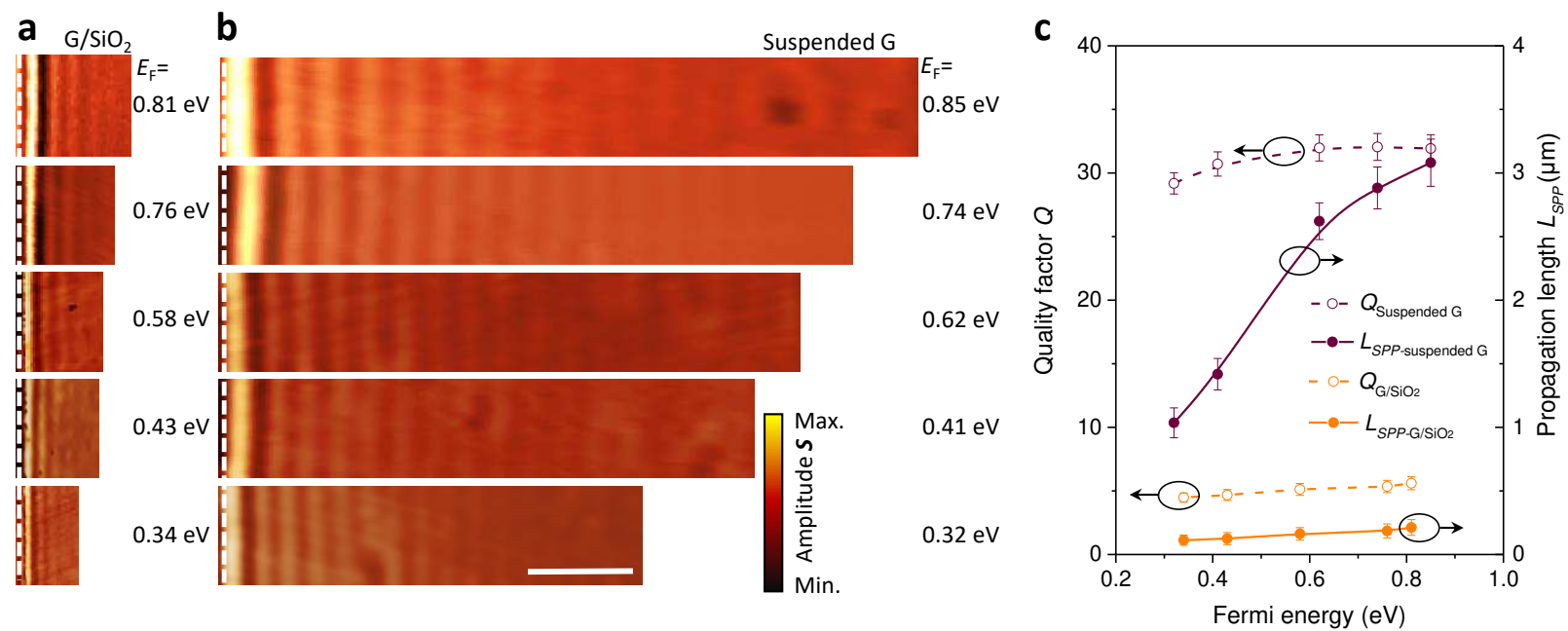

Figure 2. High-quality factor and long-distance propagation of suspended GPs. (a) and (b) Measured near-field amplitude images of $\mathrm{SiO}_{2}$-supported graphene (a) and suspended graphene (suspension height $\sim 450 \mathrm{~nm}$ ) (b) near the edge (white dashed line) for different graphene Fermi energies and fixed $\lambda_{0}=10.87 \mu \mathrm{m}\left(920 \mathrm{~cm}^{-1}\right)$. The scale bar indicates $3 \mu \mathrm{m}$. (c) Quality factor $Q$ (left axis) and propagation length $L_{\mathrm{SPP}}$ (right axis) of suspended (maroon curves) and $\mathrm{SiO}_{2}-$ supported (orange curves) GPs as a function of Fermi energy. Symbols are obtained from experimental data, while curves are a guide to the eye. Error bars indicate the $95 \%$ confidence intervals.

While in Figure 1c we have demonstrated a considerable propagation length of suspended GPs, in Figure 2a, b we compare the near-field amplitude images of plasmon propagation in suspended and $\mathrm{SiO}_{2}$-supported graphene with similar Fermi energies. The near-field images in Figure $2 \mathrm{~b}$ are flanked by the boundary (indicated by the white dashed lines) of a circular suspended region with a diameter of $30 \mu \mathrm{m}$ and a suspension height of $\sim 450 \mathrm{~nm}$. To quantify the improvement brought about by suspended graphene, we need a direct comparison of the quality factor $Q$. In momentum space, the complex, frequency-dependent plasmon wave vector is given by ${ }^{2,13} q_{p}(\omega)=q_{1}(\omega)+$ $i q_{2}(\omega)=\frac{i \kappa \omega}{2 \pi \sigma(\omega)}$, where subindices 1 and 2 indicate the real and imaginary parts, respectively, $\sigma(\omega)$ is the graphene surface conductivity, and $\kappa$ is the effective dielectric function of the graphene environment. The quality factor $Q$ is given by $Q^{-1}=\frac{q_{2}}{q_{1}} \approx \frac{\sigma_{1}}{\sigma_{2}}+\frac{\kappa_{2}}{\kappa_{1}}$, under the conditions $\left|\sigma_{2}\right| \gg\left|\sigma_{1}\right|$ and $\left|\kappa_{1}\right| \gg\left|\kappa_{2}\right|$, which are well satisfied in this study. Also, the plasmon propagation length can be expressed as $L_{S P P}=\frac{1}{2 q_{2}}=\frac{1}{4 \pi} \lambda_{p} Q$. We implement a multi-beam interference model (see Figure S7 and Supplementary Information Note 2) to analyze complex near-field amplitude images, ${ }^{30}$ from which $q_{2}(\omega)$ can be extracted through direct real-space fitting of the near-field 
signal line scans (Figure S8 and Note 2; see Supplementary Information for more details of the analysis method).

The extracted values of $Q$ and $L_{\mathrm{SPP}}$ as a function of Fermi energy (Figure 2c and S9) clearly reveal a remarkable improvement in suspended graphene samples (orange curves) over supported ones (maroon curves). The quality factor of suspended GPs varies from $Q=29$ to $Q=33$ when $E_{\mathrm{F}}$ is changed from $0.3 \mathrm{eV}$ to $0.8 \mathrm{eV}$, while in $\mathrm{SiO}_{2}$-supported plasmons $Q$ stays below 5.4. Additionally, $L_{\mathrm{SPP}}$ increases from $1.0 \mu \mathrm{m}$ to $3.1 \mu \mathrm{m}$ with increasing $E_{\mathrm{F}}$ in suspended GPs, while it is limited to the 0.1-0.2 $\mu \mathrm{m}$ range in $\mathrm{SiO}_{2}$-supported plasmons as a result of their smaller quality factor and plasmon wavelength. In principle, the plasmon wavelength of graphene in the air (dielectric constant $\varepsilon_{0} \approx 1$ ) and supported on a $\mathrm{SiO}_{2}$ substrate satisfy the relation $\lambda_{\text {suspended }} / \lambda_{\text {supported }}=\left(1+\varepsilon_{\mathrm{SiO}_{2}}\right) / 2$, where the real part of $\varepsilon_{\mathrm{SiO}_{2}}$ is 3.8 at $920 \mathrm{~cm}^{-1}{ }^{31}$ The improvement in the quality factor in suspended graphene is due to the reduced environmental losses brought about by the suspended graphene (Supplementary Information Note 1). However, the quality factor of our suspended graphene samples does not reach the estimated intrinsic limit $Q \sim 40-70$ at room temperature. ${ }^{11}$ We attribute this to impurity scattering produced by gas-molecule dopants, which provide an extra extrinsic decay channel. ${ }^{32}$ A possible mechanism could be mediated by the exchange of momentum with the graphene crystal lattice, as $\mathrm{NO}_{2}$ molecules have a size comparable to the unit cell, leading to local heating and additional plasmon damping. Nevertheless, we observe that the quality factor of suspended GPs samples is always higher than supporting GPs at room temperature. 

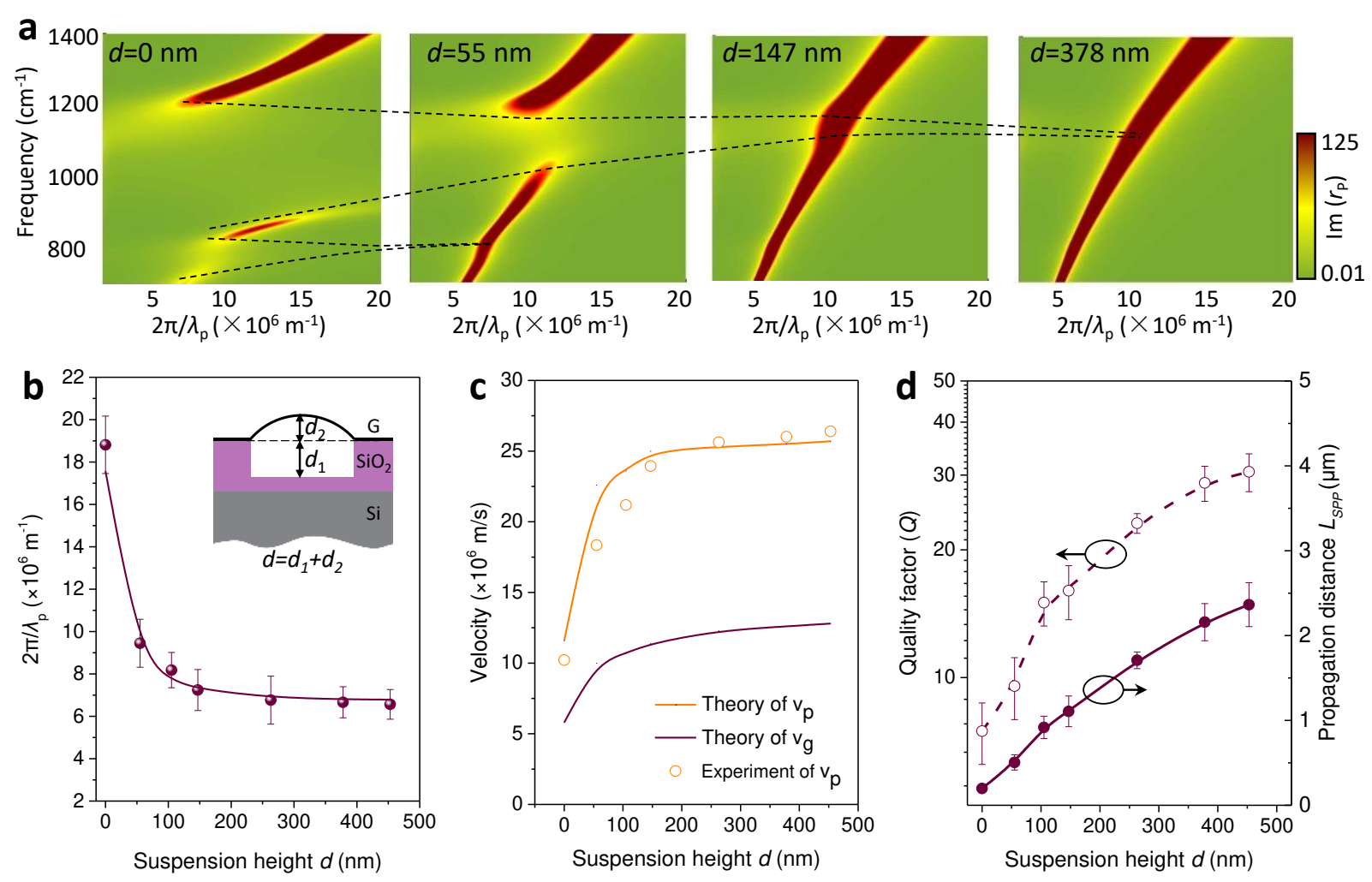

Figure 3. Active control of suspended graphene plasmons by changing the suspension height. (a) Calculated imaginary part of the loss function $\operatorname{Im}\left\{r_{\mathrm{p}}(\mathrm{q}, \omega)\right\}$ in the RPA for different suspension heights $d$, with fixed $E_{\mathrm{F}}=0.65 \mathrm{eV}$ and $\lambda_{0}=10.87 \mu \mathrm{m}\left(920 \mathrm{~cm}^{-1}\right)$. The black-dashed curves indicate the evolution of the energy splitting stemming from the strong coupling between GPs and phonon polaritons in $\mathrm{SiO}_{2}$. (b) Experimentally measured (symbols) and theoretically calculated (solid curve) plasmon wave vector as a function of the suspension height $d$ at an incident wavelength $\lambda_{0}$ $=10.87 \mu \mathrm{m}\left(920 \mathrm{~cm}^{-1}\right)$. Inset: illustration of a cross-sectional side view of the suspended graphene device. $d_{1}$ and $d_{2}$ represent the depth of the dimple and the graphene bubble's relative height, respectively. The former $\left(d_{1}\right)$ is pre-determined in the fabrication process, while $d_{2}$ can be varied in situ by controlling the gas pressure from beneath the substrate film. (c) Extracted phase (orange curves) and group (maroon curves) velocities as a function of $d$. Solid curves are theoretical predictions, while symbols are values determined from the experiment. (d) Quality factor $Q$ (left axis) and propagation length $L_{\mathrm{SPP}}$ (right axis) of GPs as a function of $d$. Symbols are obtained from experimental data, and curves indicate a guide to the eye. The error bars are from different line profiles in one scan image.

The elimination of interactions from the substrate renders a unique pure plasmonic environment, making it a unique platform for manipulating the out-of-plane interaction between intrinsic GPs and the dielectric environment. In Figure 3a, we show the calculated plasmon dispersion with different suspension heights $d$. The height $d$ between suspended graphene and the substrate is determined by the sum of the hole depth $d_{1}$ and the height of the graphene bubble $d_{2}$ relative to the 
substrate plane. The strength of long-range Fröhlich coupling between GPs and the optical phonons of the $\mathrm{SiO}_{2}$ substrate is strongest when graphene is directly on top of the substrate $(d=0)$, where the dispersion splits into three bands. ${ }^{6}$ We observe that, as $d$ increases from 0 to $453 \mathrm{~nm}$, the coupling strength gradually weakens (black-dashed curves in Figure 3a) and eventually disappears at $d=55 \mathrm{~nm}$ and $147 \mathrm{~nm}$ for the surface optical phonons at $\omega_{1}=806 \mathrm{~cm}^{-1}$ and $\omega_{2}=1168$ $\mathrm{cm}^{-1}$, respectively. In Figure S10, we show the corresponding measured near-field amplitude images with increasing suspension height $d$. The plasmon wavelength can be tuned over a wide range (from $400 \mathrm{~nm}$ to $1.2 \mu \mathrm{m}$ ) due to its strong dependence on the permittivity of the environment. Figure $3 \mathrm{~b}$ shows that, for different suspension heights $d$, the extracted plasmon wave vectors from measured near-field amplitude images (symbols) agree quite well with the calculated results (orange curve). ${ }^{33}$ Besides the significant control over the plasmon wavelength by changing the suspension height, both phase $\left(v_{\mathrm{P}}=\omega / \mathrm{q}_{1}\right)$ and group $\left(v_{\mathrm{g}}=\partial \omega / \partial \mathrm{q}_{1}\right)$ velocities sharply increase with $d$ in the $d<80 \mathrm{~nm}$ region, and they saturate at $d \sim 200 \mathrm{~nm}$, as shown in Figure 3c. Notably, $v_{\mathrm{g}}$ can vary within a range of $0.42-2.5 \times 10^{7} \mathrm{~m} / \mathrm{s}$, suggesting the possibility of broadly controlling the propagation speed of information in a graphene waveguide.

Modulation of the interaction between GPs and a substrate can also affect the plasmon quality factor. In Figure $3 \mathrm{~d}$ and $\mathrm{S} 10 \mathrm{~b}$, we show the analysis of the quality factor $Q$ and propagation length $L_{S P P}$ for different suspension heights $d$. Once graphene is detached from the substrate, the quality factor $Q$ increases dramatically due to the reduced coupling to dielectric losses from the $\mathrm{SiO}_{2}$ substrate (see detailed discussion in Figure S11 and Supplementary Information Note 3). This is consistent with the exponential decay of the electric field associated with plasmons away from the graphene plane. By computing the percentage of the near-field intensity in the proximity of graphene, we find that $65 \%$ of the mode energy $\left(\lambda_{\mathrm{p}} \approx 1.5 \mu \mathrm{m}\right.$ at a suspension height of $\left.d=453 \mathrm{~nm}\right)$ is confined within $100 \mathrm{~nm}$ above or below the graphene plane. ${ }^{11}$ We observe a twofold improvement of $Q$ when $d$ increases to $55 \mathrm{~nm}$, while a milder increase in $Q$ takes place after $d$ exceeds $100 \mathrm{~nm}$. For the achieved maximal suspension height $(d=453 \mathrm{~nm})$, the quality factor $Q$ is enhanced by about a factor of six, and the corresponding $L_{S P P}$ increases by one order of magnitude (from 0.25 to $\sim 3.0 \mu \mathrm{m}$ ) compared with the values observed in supported graphene. 

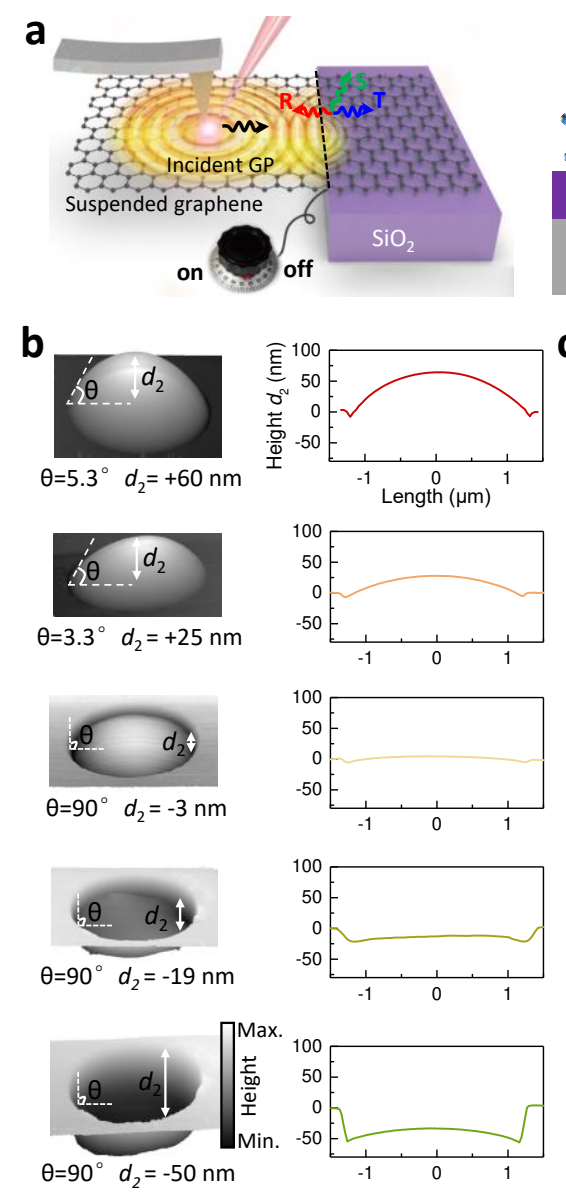
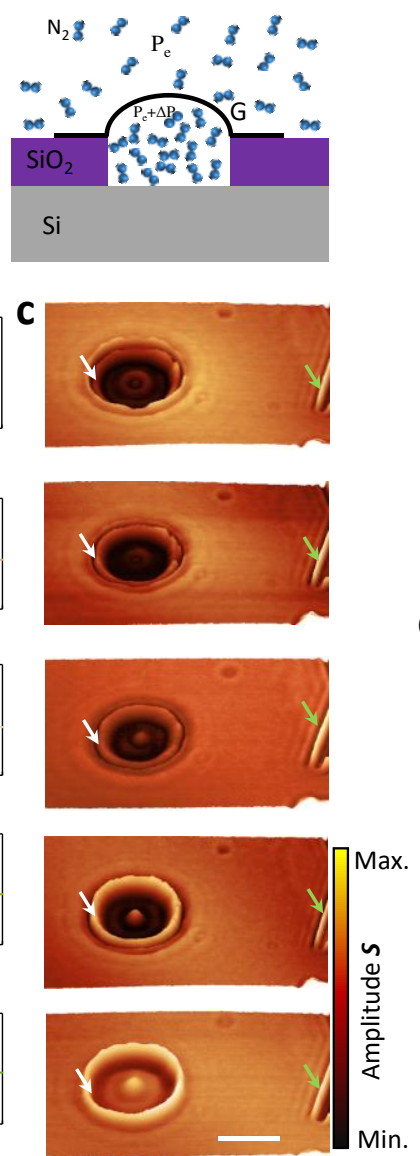

d
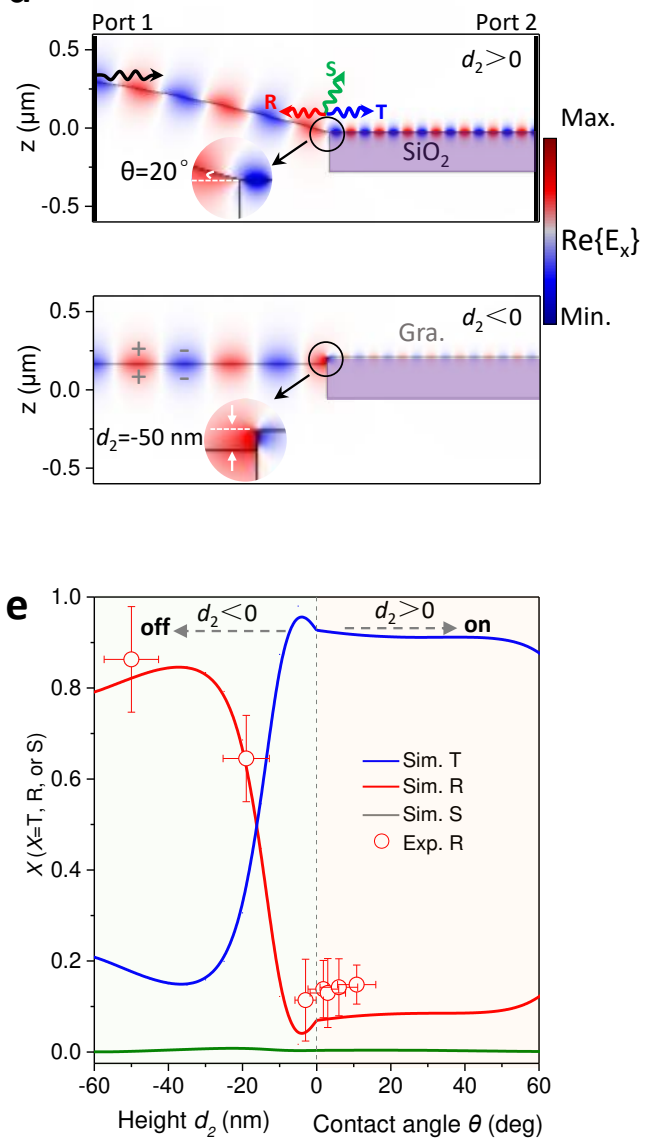

Figure 4. Plasmonic switch based on tunable control of GP transmission at air-dielectric substrate interfaces. (a) Left: a schematic of the plasmonic switch. Black, red, and blue arrows represent incident, reflected, and transmitted plasmons, respectively. The green arrow stands for plasmon scattering (out-coupling) into radiation. Right: schematic of gas diffusion into a graphene bubble from the chamber when applying very high pressure $\left(\mathrm{P}_{\mathrm{a}}\right) . \mathrm{P}_{\mathrm{e}}$ indicates the external atmospheric pressure. (b) Left: AFM topography images of suspended graphene with different heights of the graphene bubble $d_{2}$ obtained by controlling the gas pressure for fixed hole depth $d_{1}=300 \mathrm{~nm}$ (see definitions in the inset of Figure $3 \mathrm{~b}$ ). A negative value of $d_{2}$ indicates that the graphene bubble is sunken inside the substrate dimple. We use a sample with large $d_{1}$ to avoid any complications in the interpretation. Right: Height profiles of suspended graphene bubbles in the left AMF images. (c) Near-field IR images of suspended graphene, taken simultaneously with the AFM topography for the corresponding suspension heights shown in (a). The incident light wavelength is $\lambda_{0}=10.87 \mu \mathrm{m}\left(920 \mathrm{~cm}^{-1}\right)$, and the graphene Fermi energy is $0.40 \pm 0.03 \mathrm{eV}$. Since the entire sample is treated with the same gas concentration and doping time, we assume that the graphene has the same Fermi energy in the substrate and suspended regions. The bright fringes at the air-dielectric interface and natural graphene edge are indicated by white and green arrows, respectively. The scale bar indicates $2 \mu \mathrm{m}$. (d) Simulated spatial distribution of the electric field along the $x$-direction as GPs propagate from the suspended region to the region supported by the $\mathrm{SiO}_{2}$ substrate, with $d_{2}>0$ (upper part) and $d_{2}<0$ (bottom part). Inset: expanded view of the 
boundary area. (e) Plasmon reflectance (red), transmittance (blue), and scattering (green) as a function of $d_{2}$ and $\theta$. Colored curves are numerical simulations with $E_{\mathrm{F}}=0.4 \mathrm{eV}$, whereas symbols represent experimental results extracted from Figure 4b. Error bars are extracted from different line profiles in each scanned image.

Both the long-distance propagation and control of suspended GPs offer unique possibilities for studying applied aspects of graphene plasmonic. Nanoscale energy transfer and management mediated by plasmons at the interface between two media are vital for the design of nanophotonic devices. ${ }^{14,16,34}$ In Figure 4 we demonstrate that plasmon energy flow through the air-dielectric interface can be controlled by gradually changing the shape of the suspended graphene, serving as a novel plasmonic switch. Our experiment setup is illustrated in Figure 4a. Indeed, we can tune the height $d_{2}$ of a graphene bubble from $-50 \mathrm{~nm}$ (concave shape) to $+60 \mathrm{~nm}$ (convex) by changing the gas pressure, as revealed by AFM and the corresponding near-field amplitude images of the circular suspended structure shown in Figure $4 \mathrm{~b}$ and $4 \mathrm{c}$. Pronounced plasmon interference fringes at both air-dielectric interfaces (indicated by a white arrow) and natural graphene edges (indicated by a green arrow) can be observed in Figure 4c, which suggests that plasmons can be efficiently reflected by the air-dielectric interface, besides the well-known total reflection at the natural graphene edges. ${ }^{14}$ The reflectance coefficient can be estimated as $r=\left(S_{\text {dielectric- }} S_{\text {sheet1 } 1}\right) /\left(S_{\text {edge- }}\right.$ $S_{\text {sheet2), }}{ }^{14,35}$ where $S_{\text {dielectric }}, S_{\text {edge }}, S_{\text {sheet1 }}$, and $S_{\text {sheet2 represent near-field signals of the bright fringe }}$ at the air-dielectric interface, the bright fringe at the natural graphene edge, and from the background of suspended ( $\left.S_{\text {sheet1 }}\right)$ and supported ( $\left.S_{\text {sheet2 }}\right)$ graphene, respectively (Figure S12). Since the near-field amplitude $S$ tracks variations in the local electric field between tip and graphene, the plasmon energy scales as the square of $S$, and then the reflectance can be evaluated as $R=|r|^{2}$. Figure $4 d$ illustrates the spatial distributions of the real part of the electrical field $\operatorname{Re}\left\{E_{x}\right\}$ along the horizontal direction for the transmission of GPs from suspended to $\mathrm{SiO}_{2}$-supported graphene regions, where a plasmonic impedance mismatch between the two regions appears due to the abrupt change of substrate permittivity and graphene morphology at the interface. Note that both the contact angle $\theta$ and the step height $d_{2}$ influence the plasmonic impedance mismatch and further affect the plasmonic transmission (Figure S13).

When the suspended graphene is above the substrate surface (i.e., $d_{2}>0$, the upper part of Figure $4 \mathrm{~d}$ ), it is convenient to exploit the contact angle $\theta$ (determined by the value of $d_{2}$ and the graphene morphology) to describe the reflection at the interface due to a different graphene morphology 
compared with the situation in which $d_{2}<0$. When $\theta$ gradually increases from 0 to $45^{\circ}$, the reflectance gradually increases from $6.5 \%$ to $8.6 \%$. However, when the graphene is below the substrate surface in the suspended region (i.e., $d_{2}<0$, bottom part of Figure $4 \mathrm{~d}$ ), graphene attaches to the sidewall of the dimple due to van der Waals attraction, and the contact angle $\theta$ stays at $90^{\circ}$, whereas $d_{2}$ mainly determines the reflection. As $d_{2}$ increases from $-50 \mathrm{~nm}$ to $-3 \mathrm{~nm}$, the fringe contrast in the suspended graphene region gradually decreases, indicating a reduced reflectance at the air-dielectric interface. We experimentally observe that the reflectance is close to $90 \%$ when $d_{2}$ decreases to $-50 \mathrm{~nm}$ with $E_{\mathrm{F}} \approx 0.4 \mathrm{eV}$ (Figure $4 \mathrm{e}$, symbols).

The minimum reflectance is $6.5 \%$ if the graphene bubble is nearly flat $\left(d_{2}=0\right.$, i.e., co-planar with the substrate surface). Since almost no free radiation is emitted in the scattering process under these conditions (green curve in Figure $4 \mathrm{e}$ ), ${ }^{36}$ the maximum energy crossing the air-dielectric interface can be considered to be $93.5 \%$. By analogy to electronic devices, we can define the ratio between the maximum and minimum energies passing across the interface as the switch ratio of plasmonic devices $;^{37}$ the observed switch ratio reaches $>14$ by in situ changing the shape of suspended graphene. Simultaneously, the plasmon wavelengths are very different at the two sides $\left(\lambda_{\mathrm{p}} \approx 530 \mathrm{~nm}\right.$ at the left in the suspended graphene region, and $\lambda_{\mathrm{p}} \approx 170 \mathrm{~nm}$ at the right in the $\mathrm{SiO}_{2-}$ supported area, as shown in Figure 4d). Therefore, the device serves as an efficient plasmon wavelength convertor.

It is worth noting that a suspended graphene electromechanical system driven by gate voltage can be used to control the reflection actively. As a thin atomic membrane with low lateral stiffness, the suspended graphene can be ideal for electromechanical devices to control its morphology. Indeed, suspended graphene can be gradually sucked into the holes with a vertical displacement under a transverse electric field. The height of suspended graphene relative to the substrate can be tuned from $+5 \mathrm{~nm}$ to $-26 \mathrm{~nm}$ with a gate voltage from 0 to $30 \mathrm{~V}$ (Figure S14). With a continuous increase of the gate voltage, the difference of height and the Fermi level on both sides can be exacerbated at the same time, which further promotes the reflection of plasmons at the boundary. As shown in Figure S14, plasmons reflection at the dielectric boundary increases with gate voltages, from $23 \%$ to $70 \%$. Other controllable parameters, such as the graphene Fermi energy and the incident light frequency, can also offer versatile methods for tunability (Figure S15, 16). 
To conclude, we demonstrate that high-frequency propagating plasmons $\left(\sim 1400 \mathrm{~cm}^{-1}\right)$ can be excited in suspended monolayer graphene with the ultrahigh doping level (up to $0.9 \mathrm{eV}$ ) introduced through gas molecule adsorption. In particular, high-quality factor $(Q=33)$ and long propagation length $\left(L_{\mathrm{SPP}}>3 \mu \mathrm{m}\right)$ in plasmons evolving in tailored suspended graphene at room temperature are also achieved by eliminating interactions from the substrate. Besides, suspended graphene provides a unique pure plasmonic platform, this grants us a better understanding and tuning of the interaction physics of the extrinsic environment with GPs. Thus, by in situ changing the suspension height of graphene, we have implemented an unprecedentedly efficient manipulation of the plasmon wavelength, propagation length, and phase/group velocity. We have further presented an effective route to fully control the plasmonic energy transmission at the air-dielectric hole-edge interface by applying electromechanical concepts to plasmonic devices, in which the suspended graphene can be deformed by a gate voltage or varying gas pressure. Furthermore, we discuss how the deformation can be tailored to create a plasmonic switch (by gas) and transistor (by a gate) to achieve near-unity-order manipulation of plasmonic energy flow. The combination of micrometerpropagation and energy manipulation can be applicable to design other advanced plasmonic devices, such as plasmonic resonators, filters, and modulators, which are pivotal to energy flows in ultra-compact integrated plasmonic circuits.

\section{Methods}

\section{Nanofabrication of suspended GP devices.}

Circular holes with sizes ranging from $30 \mathrm{~nm}$ to $50 \mu \mathrm{m}$ were patterned on a $300 \mathrm{~nm} \mathrm{SiO}_{2} / 500$ $\mu \mathrm{m}$ Si substrate using $100 \mathrm{kV}$ electron-beam lithography (EBL) (Vistec 5000+ES, Germany) on approximately $350 \mathrm{~nm}$ of ZEP520A electron beam lithography resist. The hole arrays were etched by $\mathrm{C}_{4} \mathrm{~F}_{8}$ and $\mathrm{SF}_{6}$ gases (North Microelectronics, DSE200). Then, the resist layer was dissolved with butanone and the whole wafer was cleaned with isopropyl alcohol. For holes that were deeper than $300 \mathrm{~nm}$, we used plasma-enhanced chemical vapor deposition (PECVD) to grow a 300-nmthick layer of $\mathrm{SiO}_{2}$ in the hole to cover the silicon substrate. The remaining residues on the silicon oxide surface were removed by oxygen plasma cleaning with $5 \mathrm{~Pa}$ and $80 \mathrm{~W}$ for $20 \mathrm{~min}$. Graphite flakes were then mechanically deposited onto the substrate. Optical microscopy was used to identify monolayer graphene sheets, and their layer numbers were further confirmed by Raman spectroscopy. To control the shape of suspended graphene, the samples were placed into the chamber with $\mathrm{N}_{2}$ to create a pressure difference $(\Delta P)$ between the inside and outside of the suspended graphene membrane. $\Delta P$ was utilized as a controlling load to change the suspension height based on the well-established gas diffusion method according to $\Delta P=\left(P_{a} \mathrm{~V}_{0} /\left(\mathrm{V}_{0}+V_{s}\right)\right)-\mathrm{P}_{\mathrm{e}}{ }^{38}$ where $P_{a}$ and $P_{e}$ are the applied and external atmosphere pressures; and $V_{0}$ and $V_{s}$ are the volumes of the hole and suspended graphene bubble, respectively. A schematic diagram of the gas diffusion process is shown in Figure 4a. Following Hencky's solution, the pressure difference across the 
membrane and the maximum deflection at the center of the suspended graphene hole follows a simple relation: $\Delta P=\operatorname{kEt}\left(d_{2}^{3} / \mathrm{r}^{4}\right)$, where $\mathrm{k}$ is a dimensionless coefficient, $\mathrm{E}$ is Young's modulus, $\mathrm{t}$ is the thickness of graphene, $d_{2}$ is the maximum deflection, and $r$ is the circular radius of the suspended graphene region. Our experimental parameters for bubble filling are shown in Figure $\mathrm{S} 17$. Then, the samples were transferred to another chamber with $\mathrm{NO}_{2}$ gas molecules to tune the Fermi energy of graphene. $\mathrm{HNO}_{3}$ vapor was also used to dope the graphene and achieve higher carrier density. We verified that heating the doped samples produced a large decrease in doping.

\section{Near-Field Optical Microscopy Measurements.}

Near-field results were measured using a scattering SNOM setup (Neaspec GmbH) equipped with wavelength-tunable lasers (between 900 and $2000 \mathrm{~cm}^{-1}$ ). The spot sizes of the Mid-IR beam under the AFM tip were made $\sim 30 \mu \mathrm{m}$ in lateral size to cover the large area of suspended graphene, thus facilitating tip and edge launching of GPs used in this research. The probes were commercially available AFM tips metalized and having an apex radius of $\sim 25 \mathrm{~nm}$ (Nanoworld). The tip-tapping frequency and amplitudes were $\sim 270 \mathrm{kHz}$ and $\sim 30-50 \mathrm{~nm}$, respectively. The near-field amplitude images were obtained from the third-order demodulated harmonic of the near-field amplitude, which resulted in massive suppression of background noise.

\section{Theoretical calculations and electromagnetic simulations.}

The dispersion relation of GPs can be obtained from the poles in the imaginary part of the Fresnel reflection coefficient $r_{p}(q, \omega)$, defined by the ratio between reflected and incident field amplitudes at the air/graphene/substrate interface, $r_{p}(q, \omega)=\frac{\varepsilon_{1} k_{0}-\varepsilon_{0} k_{1}+4 \pi k_{0} k_{1} \sigma / \omega}{\varepsilon_{1} k_{0}+\varepsilon_{0} k_{1}+4 \pi k_{0} k_{1} \sigma / \omega}$, where $\varepsilon_{0}$ is the dielectric constant of air, $\varepsilon_{1}$ is the complex dielectric function of $\mathrm{SiO}_{2}, \sigma=\sigma(q, \omega)$ is the inplane optical conductivity of graphene, and $k_{0}$ and $k_{1}$ represent the out-of-plane light-wavevector components in air and $\mathrm{SiO}_{2}$, respectively. ${ }^{26}$ The permittivities of silicon dioxide and silicon at $10.87 \mu \mathrm{m}$ light wavelength are $\varepsilon\left(\mathrm{SiO}_{2}\right)=3.8$ and $\varepsilon(\mathrm{Si})=8.9$, respectively. ${ }^{39}$ For suspended graphene, $r_{p}(q, \omega)=\frac{4 \pi k_{0}^{2} \sigma / \omega}{2 \varepsilon_{0} k_{0}+4 \pi k_{0}^{2} \sigma / \omega}$, In general, the graphene conductivity is a function of photon frequency $\omega$, Fermi energy $E_{F}$, inelastic relaxation time $\tau$, and temperature $T$. Under the conditions $k_{B} T \ll \hbar \omega$ and $k_{B} T<<\left|E_{\mathrm{F}}\right|$, the conductivity $\sigma$ can be modeled in the local limit of the RPA $^{40}$ and decomposed into intraband and interband contributions as $\sigma=\sigma_{\text {intra }}+$ $\sigma_{\text {inter }}$, where $\sigma_{\text {intra }}=\frac{e^{2}}{\pi \hbar^{2}} \frac{i E_{F}}{\omega+i \tau^{-1}}, \sigma_{\text {inter }}=i \frac{e^{2}}{4 \pi \hbar} \ln \left(\frac{2\left|E_{F}\right|-\hbar\left(\omega+i \tau^{-1}\right)}{2\left|E_{F}\right|+\hbar\left(\omega+i \tau^{-1}\right)}\right), \quad e$ is the electron charge, and $\hbar$ is the reduced Planck constant. The relaxation time $\tau=\mu E_{F} / e v_{F}^{2}$ depends on the graphene Fermi velocity $v_{F}=c / 300$ and the carrier mobility $\mu$.

The simulated results were shown in Figure 1, 4, and S5 were obtained using a Finite Elements Method package (COMSOL). In the simulation, the graphene layer was assigned a finite thickness $t_{\mathrm{g}}=1 \mathrm{~nm}$ and an effective dielectric function $\varepsilon=1+\mathrm{i} 4 \pi \sigma /\left(\omega t_{\mathrm{g}}\right)$. For the near-field image simulations in Figure 1d and S5, the graphene was modeled as a transition interface with the above dielectric properties. We approximated the tip by a vertical point dipole source featuring an oscillating dipole placed at the distance of $60 \mathrm{~nm}$ from the suspended graphene surface. The model was designed as an axisymmetric circular aperture structure, and we calculated the out-of-plane electric near-field component $E_{z}$ as a function of the dipole position along a radial line crossing the center of the circular suspended graphene region by exploiting the axisymmetry. The $E_{z}$ distribution was then expanded into a $2 \mathrm{D}$ axisymmetric plot. In the simulation, the contact angle $\theta$ was determined by the suspension height $d_{2}$ through a function fitted from experimental 
measurements (Figure 4b).

In the simulations of GP transmission in Figure 4, the GPs were launched from a port to the left and then propagated along with the graphene from the suspended region to the $\mathrm{SiO}_{2}$-supported area toward a port to the right. The simulation was performed on half the suspended graphene region due to the symmetrical structure of the samples used in the experiments. The two numerical waveguide ports were placed at the ends of graphene to produce GPs. A GP impinging from the left at the interface could be reflected (GP moving to the left), transmitted (GP moving to the right), or out-coupled as a free-space IR photon. When the suspended graphene was above the substrate surface (i.e., $d_{2}>0$ ), the structure of suspended graphene was matched through a simple deflection profile as a result of the competition between the elastic energy of the deformed suspended graphene and the uniform force associated with gas pressure, as well as the adhesion energy (between graphene and the substrate), which can be fitted as $y(x)=d_{2}\left(1-x^{2} /\right.$ $a^{2}$ ) with $95 \%$ confidence level, ${ }^{41}$ where $d_{2}$ is the height of the center point of the suspended graphene to the substrate surface, and $a=1.25 \mu \mathrm{m}$ is the radius of the hole. We set the center point of suspended graphene to $x=0$ ( $x$ was along the radial direction). The relationship between the contact angle $\theta$ and the height $d_{2}$ was extracted by taking the derivative at the edge of suspended graphene $(x=a): \tan (\theta)=2 d_{2} / a$, where $\theta$ is in the $[0, \pi / 2)$ range. The inelastic loss of GPs was not considered throughout the process, and the graphene thickness was assumed to be $1 \mathrm{~nm}$. The reflectance $R$ at the interface was defined as the ratio of the reflected GP energy (propagating to

the left) to the incident GP energy (propagating from the left to the interface): $R=\frac{\left|E_{R}\right|^{2}}{\left|E_{\text {in }}\right|^{2}}$. We note that in our simulations the out-coupling channel produced only minor corrections.

\section{Acknowledgments}

This work was supported by the National Natural Science Foundation of China (Grant Nos. 51902065, 51925203, 51372045, 11504063, and 11674073), National Key Research and Development Program of China (Grant No. 2020YFB2205700, 2015CB932400), Beijing Municipal Natural Science Foundation (Grant No. 20B20253), the National Key Research and Development Program of China (Grant No. 2016YFA0201600), the the key program of the bureau of Frontier Sciences and Education Chinese Academy of Sciences (Grant No. QYZDBSSWSLH021). F.J.G.A. acknowledges the ERC (Advanced Grant 789104- eNANO), the Spanish MINECO (MAT2017-88492-R and SEV2015-0522), and the European Commission (Graphene Flagship Grant No. 696656). Academy of Finland (Grant Nos. 276376, 284548, 295777, 304666, 312297, 312551, and 314810), TEKES (OPEC), and the European Union's Seventh Framework Programme (REA grant agreement No. 631610).

\section{Data availability}

The data that support the findings of this study are available from the corresponding author upon reasonable request.

\section{Author contributions}

Q.D. and H.H. conceived the idea. Q.D., H.H., and J.A. led the experiments. R.Y. and F.J.G.A. developed the theory. G.X., H.H., and H.T. performed the simulation. H.H. designed the structures and prepared the samples. H.H. and H.D. performed the near-field measurements. H.H., R.Y., and M.L. analyzed the data, and all authors discussed the results. H.H., R.Y., M.L., and F.J.G.A. wrote the manuscript, with input and comments from all authors. 
Competing interests The authors declare no competing interests.

\section{Reference}

1. Ju L, Geng B, Horng J, Girit C, Martin M, Hao Z, et al. Graphene plasmonics for tunable terahertz metamaterials. Nat Nanotech 2011, 6(10): 630-634.

2. Fei Z, Rodin AS, Andreev GO, Bao W, McLeod AS, Wagner M, et al. Gate-tuning of graphene plasmons revealed by infrared nano-imaging. Nature 2012, 487(7405): 82-85.

3. Chen JN, Badioli M, Alonso-Gonzalez P, Thongrattanasiri S, Huth F, Osmond J, et al. Optical nano-imaging of gate-tunable graphene plasmons. Nature 2012, 487(7405): 77-81.

4. Ni GX, WangL, Goldflam MD, WagnerM, FeiZ, McLeod AS, et al. Ultrafast optical switching of infrared plasmon polaritons in high-mobility graphene. Nat Photon 2016, 10(4): 244-247.

5. Woessner A, Lundeberg MB, Gao Y, Principi A, Alonso-González P, Carrega M, et al. Highly confined low-loss plasmons in graphene-boron nitride heterostructures. Nat Mater 2015, 14(4): 421-425.

6. Yan H, Low T, Zhu W, Wu Y, Freitag M, Li X, et al. Damping pathways of mid-infrared plasmons in graphene nanostructures. Nat Photon 2013, 7(5): 394.

7. Chakraborty S, Marshall OP, Folland TG, Kim Y-J, Grigorenko AN, Novoselov KS. Gain modulation by graphene plasmons in aperiodic lattice lasers. Science 2016, 351(6270): 246-248.

8. Cox JD, Javier García de Abajo F. Electrically tunable nonlinear plasmonics in graphene nanoislands. Nat Commun 2014, 5.

9. Alonso-González P, Nikitin AY, Golmar F, Centeno A, Pesquera A, Vélez S, et al. Controlling graphene plasmons with resonant metal antennas and spatial conductivity patterns. Science 2014, 344(6190): 1369-1373.

10. Freitag M, Low T, Zhu W, Yan H, Xia F, Avouris P. Photocurrent in graphene harnessed by tunable intrinsic plasmons. Nat Commun 2013, 4(1): 1-8.

11. Rodrigo D, Limaj O, Janner D, Etezadi D, De Abajo FJG, Pruneri V, et al. Mid-infrared plasmonic biosensing with graphene. Science 2015, 349(6244): 165-168.

12. Koppens FH, Chang DE, Garcia de Abajo FJ. Graphene plasmonics: a platform for strong light-matter interactions. Nano Lett 2011, 11(8): 3370-3377.

13. Ni GX, McLeod AS, Sun Z, Wang L, Xiong L, Post KW, et al. Fundamental limits to graphene plasmonics. Nature 2018, 557(7706): 530-533.

14. Jiang L, Shi Z, Zeng B, Wang S, Kang J-H, Joshi T, et al. Soliton-dependent plasmon reflection at bilayer graphene domain walls. Nat Mater 2016, 15(8): 840-844.

15. Jiang BY, Ni GX, Pan C, Fei Z, Cheng B, Lau CN, et al. Tunable Plasmonic Reflection by Bound 1D Electron States in a 2D Dirac Metal. Phys Rev Lett 2016, 117(8): 086801.

16. Fei Z, Rodin A, Gannett W, Dai S, Regan W, Wagner M, et al. Electronic and plasmonic phenomena at graphene grain boundaries. Nat Nanotech 2013, 8(11): 821-825.

17. Jiang B-Y, Mele EJ, Fogler MM. Theory of plasmon reflection by a 1D junction. Opt Express 2018, 26(13): 17209-17226.

18. Bolotin KI, Sikes K, Jiang Z, Klima M, Fudenberg G, Hone J, et al. Ultrahigh electron mobility in suspended graphene. Solid State Commun 2008, 146(9-10): 351-355.

19. Du X, Skachko I, Barker A, Andrei EY. Approaching ballistic transport in suspended graphene. Nat Nanotech 2008, 3(8): 491-495.

20. Prechtel L, Song L, Schuh D, Ajayan P, Wegscheider W, Holleitner AW. Time-resolved ultrafast photocurrents and terahertz generation in freely suspended graphene. Nat Commun 2012, 3(1): 1-7. 
21. Kim YD, Kim H, Cho Y, Ryoo JH, Park C-H, Kim P, et al. Bright visible light emission from graphene. Nat Nanotech 2015, 10(8): 676.

22. Chen C, Rosenblatt S, Bolotin KI, Kalb W, Kim P, Kymissis I, et al. Performance of monolayer graphene nanomechanical resonators with electrical readout. Nat Nanotech 2009, 4(12): 861.

23. Seol JH, Jo I, Moore AL, Lindsay L, Aitken ZH, Pettes MT, et al. Two-dimensional phonon transport in supported graphene. Science 2010, 328(5975): 213-216.

24. Schedin F, Geim AK, Morozov SV, Hill E, Blake P, Katsnelson M, et al. Detection of individual gas molecules adsorbed on graphene. Nat Mater 2007, 6(9): 652-655.

25. Farmer DB, Avouris P, Li Y, Heinz TF, Han S-J. Ultrasensitive Plasmonic Detection of Molecules with Graphene. ACS Photon 2016, 3(4): 553-557.

26. Fei Z, Andreev GO, Bao W, Zhang LM, McLeod AS, Wang C, et al. Infrared nanoscopy of Dirac plasmons at the graphene-SiO2 interface. Nano Lett 2011, 11(11): 4701-4705.

27. Nikitin A, Alonso-González P, Vélez S, Mastel S, Centeno A, Pesquera A, et al. Real-space mapping of tailored sheet and edge plasmons in graphene nanoresonators. Nat Photon 2016, 10(4): 239.

28. Dai S, Ma Q, Yang Y, Rosenfeld J, Goldflam MD, McLeod A, et al. Efficiency of launching highly confined polaritons by infrared light incident on a hyperbolic material. Nano Lett 2017, 17(9): 5285-5290.

29. De Abajo FG. Colloquium: Light scattering by particle and hole arrays. Rev Mod Phys 2007, 79(4): 1267.

30. Liao B, Guo X, Hu D, Zhai F, Hu H, Chen K, et al. A Multibeam Interference Model for Analyzing Complex Near-Field Images of Polaritons in 2D van der Waals Microstructures. $A d v$ Funct Mater 2019, 29(42): 1904662.

31. Pellegrino FMD, Angilella GGN, Pucci R. Linear response correlation functions in strained graphene. Phys Rev B 2011, 84(19).

32. Novko D. Dopant-induced plasmon decay in graphene. Nano Lett 2017, 17(11): 6991-6996.

33. Hwang E, Sarma SD. Dielectric function, screening, and plasmons in two-dimensional graphene. Phys Rev B 2007, 75(20): 205418.

34. Garcia-Pomar JL, Nikitin AY, Martin-Moreno L. Scattering of graphene plasmons by defects in the graphene sheet. ACS Nano 2013, 7(6): 4988-4994.

35. Chen J, Nesterov ML, Nikitin AY, Thongrattanasiri S, Alonso-González P, Slipchenko TM, et al. Strong plasmon reflection at nanometer-size gaps in monolayer graphene on SiC. Nano Lett 2013, 13(12): 6210-6215.

36. Chaves A, Amorim B, Bludov YV, Gonçalves PAD, Peres N. Scattering of graphene plasmons at abrupt interfaces: An analytic and numeric study. Phy Rev B 2018, 97(3): 035434.

37. Slipchenko TM, Nesterov ML, Hillenbrand R, Nikitin AY, Martín-Moreno L. Graphene plasmon reflection by corrugations. ACS Photon 2017, 4(12): 3081-3088.

38. Koenig SP, Boddeti NG, Dunn ML, Bunch JS. Ultrastrong adhesion of graphene membranes. Nat Nanotech 2011, 6(9): 543.

39. Edward DP, Palik I. Handbook of optical constants of solids. Academic, Orlando, Florida; 1985.

40. Chen P-Y, Alù A. Atomically Thin Surface Cloak Using Graphene Monolayers. ACS nano 2011, 5(7): 5855-5863.

41. Sanchez DA, Dai Z, Wang P, Cantu-Chavez A, Brennan CJ, Huang R, et al. Mechanics of spontaneously formed nanoblisters trapped by transferred 2D crystals. PNAS, 2018, 115(31): 7884-7889. 


\section{Figures}

a

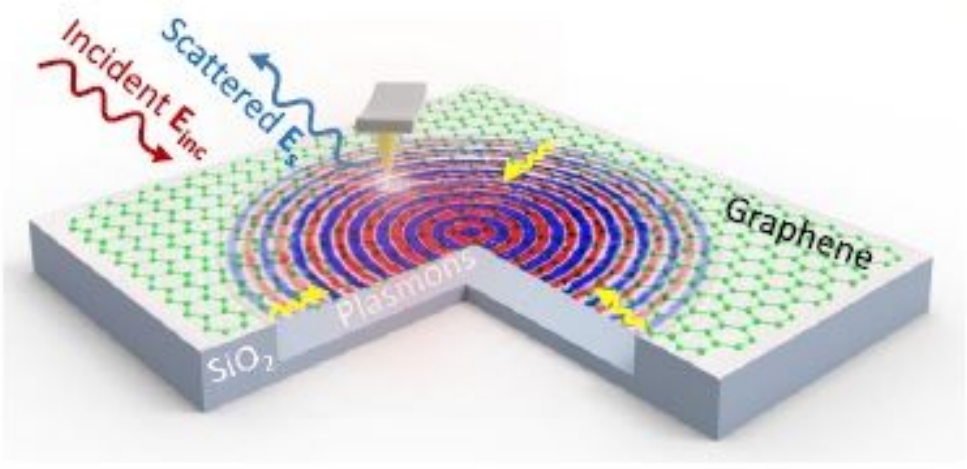

C

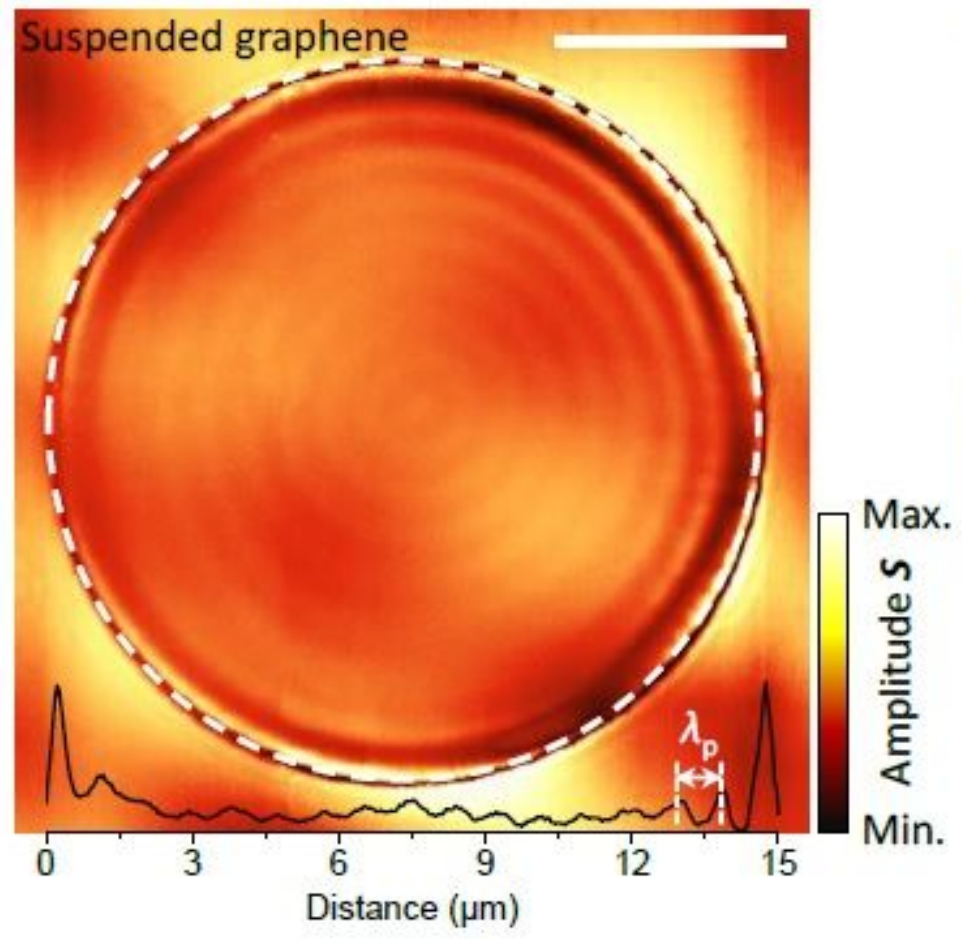

b

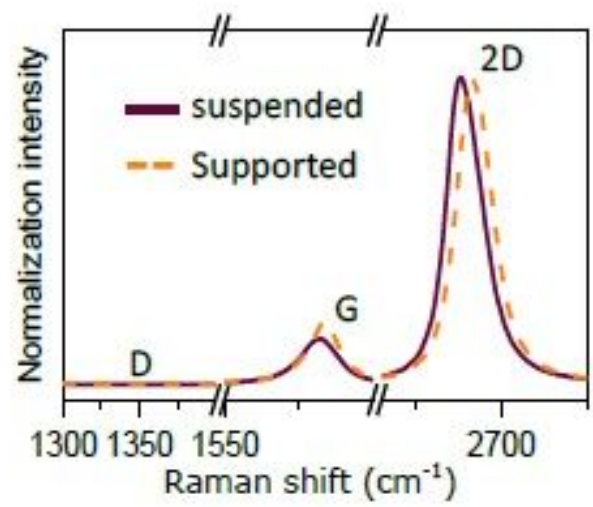

d

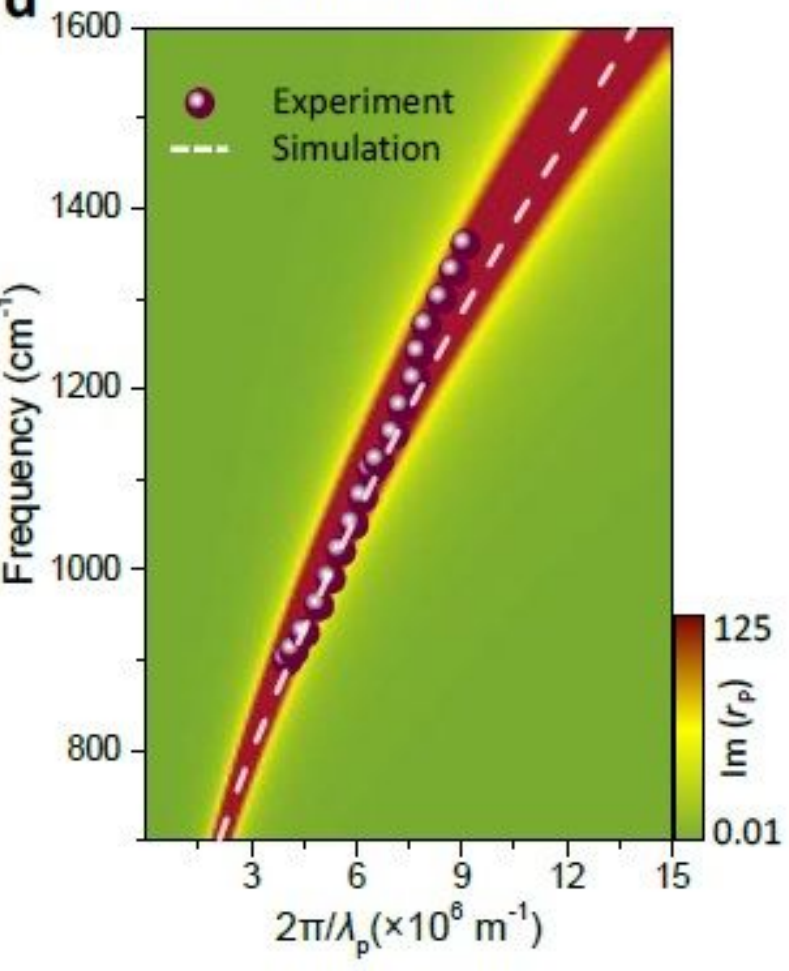

\section{Figure 1}

Plasmonic response and intrinsic dispersion in suspended graphene structures. (a) Sketch of experimental arrangement for launching and detecting plasmons propagating in suspended graphene. The tip and sample are illuminated with a focused infrared light (with wavelength $\lambda 0$ and field Einc), which can induce dipoles around the hole edge, thus efficiently exciting the graphene plasmons (yellow arrows). The tip subsequently scatters the plasmon, and a distant detector measures the scattered field ES. Note that we use defocus processing to enlarge the spot size to 30 microns and enhance the surrounding edge launching efficiency further. (b) Raman spectra of suspended and SiO2-supported regions of high-quality undoped graphene. (c) Near-field optical signal at $\lambda 0=10.87 \mu \mathrm{m}(920 \mathrm{~cm}-1)$ light wavelength from a two-dimensional scan across the suspended graphene area with a circular diameter $\Phi=15 \mu \mathrm{m}$ and a suspension height $\sim 450 \mathrm{~nm}$. The interface between suspended and supported graphene 
regions is marked with a white dashed circle. The black line at the bottom shows the near-field profile along with a horizontal cut through the center. The graphene Fermi energy is EF $\sim 0.68 \mathrm{eV}$. The scale bar indicates $5 \mu \mathrm{m}$. (d) Experimentally measured (symbols, from another device with $\Phi=2.5 \mu \mathrm{m}$ and $E F \sim 0.9$ eV, shown in Figure S4) and simulated (white dashed curve, details in Methods) dispersion of suspended GPs. The loss function $\operatorname{Im}\{r p(q, \omega)\}$ calculated from the reflection coefficient $r p$ in the random-phase approximation (RPA, details in Methods) is shown as a pseudo-color-plot background.
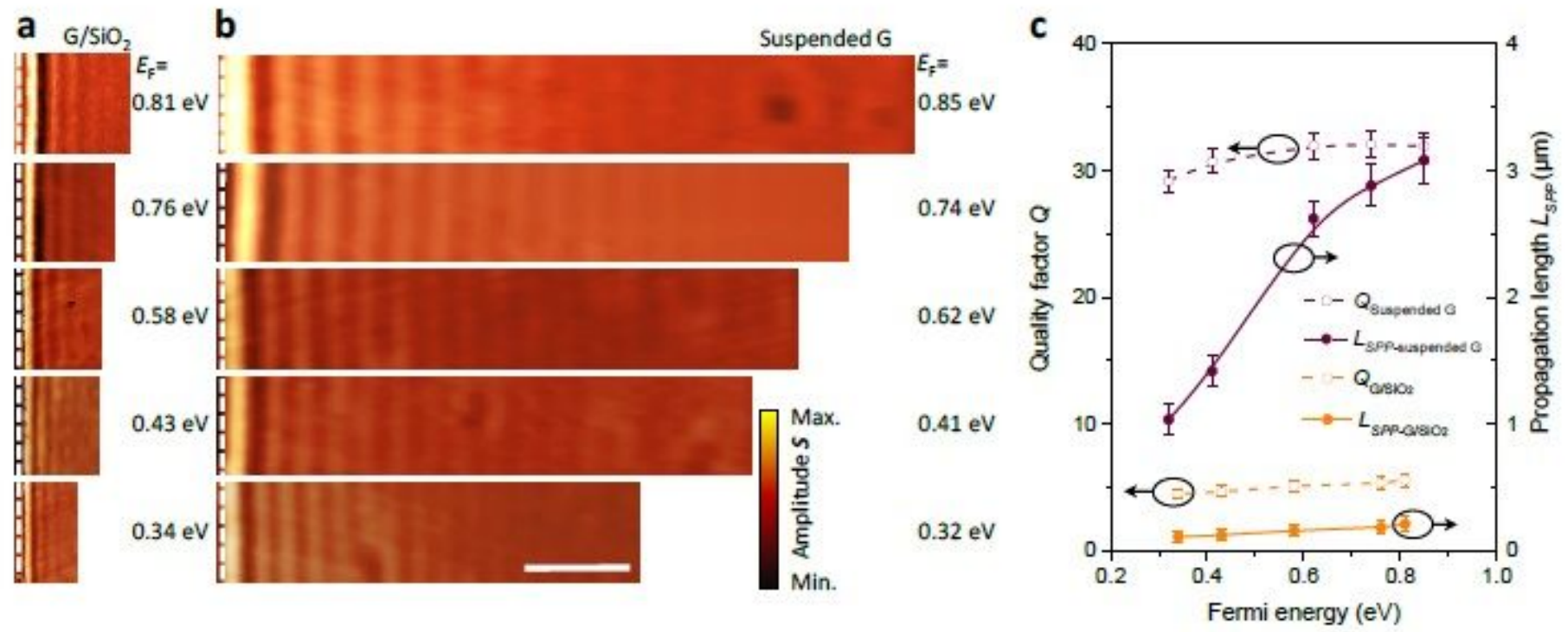

\section{Figure 2}

High-quality factor and long-distance propagation of suspended GPs. (a) and (b) Measured near-field amplitude images of SiO2-supported graphene (a) and suspended graphene (suspension height 450 $\mathrm{nm}$ ) (b) near the edge (white dashed line) for different graphene Fermi energies and fixed $\lambda 0=10.87 \mu \mathrm{m}$ $(920 \mathrm{~cm}-1)$. The scale bar indicates $3 \mu \mathrm{m}$. (c) Quality factor Q (left axis) and propagation length LSPP (right axis) of suspended (maroon curves) and SiO2-supported (orange curves) GPs as a function of Fermi energy. Symbols are obtained from experimental data, while curves are a guide to the eye. Error bars indicate the $95 \%$ confidence intervals. 

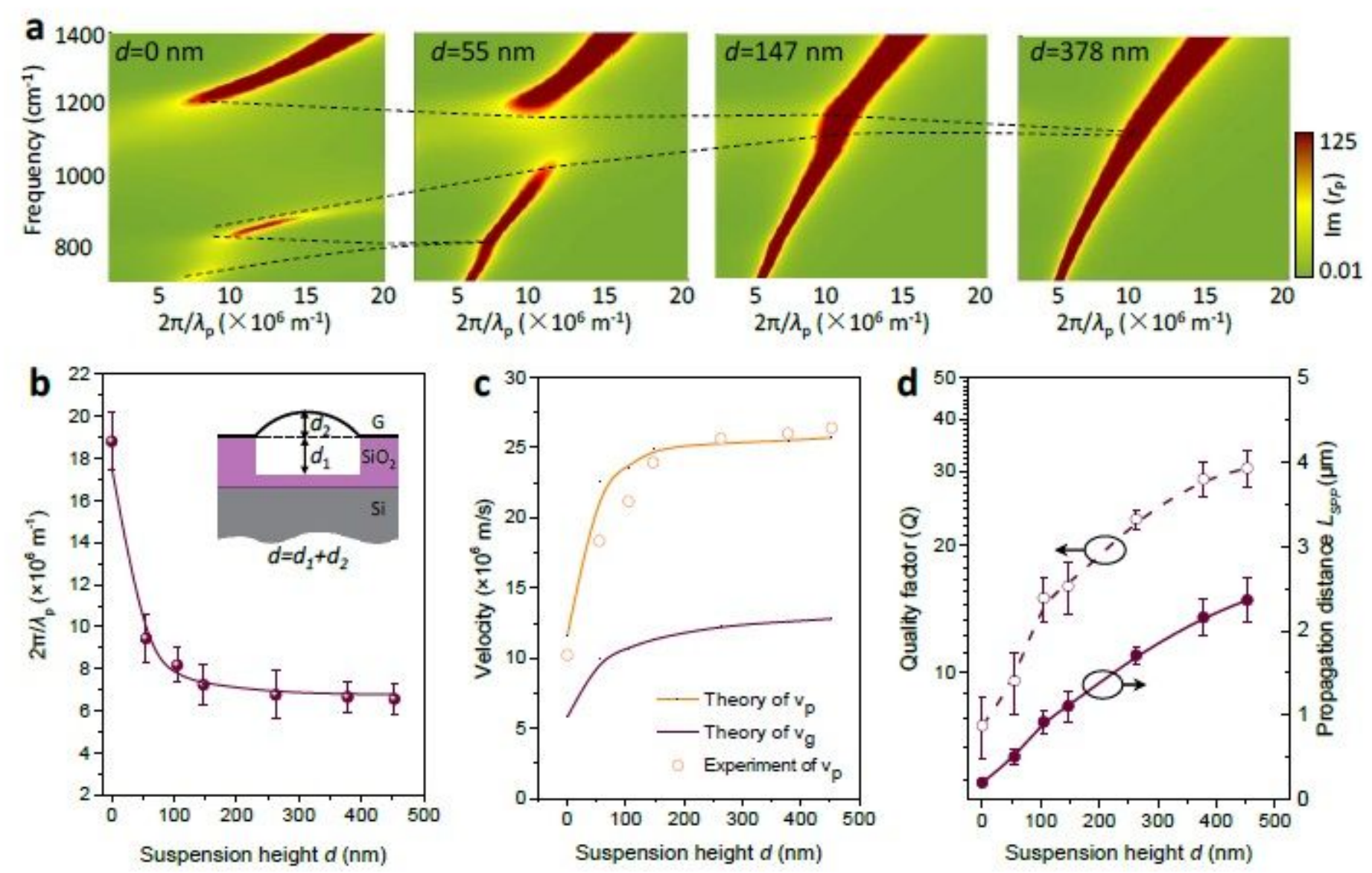

Figure 3

Active control of suspended graphene plasmons by changing the suspension height. (a) Calculated imaginary part of the loss function $\operatorname{Im}\{r p(q, \omega)\}$ in the RPA for different suspension heights $d$, with fixed $\mathrm{EF}=0.65 \mathrm{eV}$ and $\lambda 0=10.87 \mu \mathrm{m}(920 \mathrm{~cm}-1)$. The black-dashed curves indicate the evolution of the energy splitting stemming from the strong coupling between GPs and phonon polaritons in SiO2. (b) Experimentally measured (symbols) and theoretically calculated (solid curve) plasmon wave vector as a function of the suspension height $d$ at an incident wavelength $\lambda 0=10.87 \mu \mathrm{m}(920 \mathrm{~cm}-1)$. Inset: illustration of a cross-sectional side view of the suspended graphene device. $\mathrm{d} 1$ and $\mathrm{d} 2$ represent the depth of the dimple and the graphene bubble's relative height, respectively. The former (d1) is predetermined in the fabrication process, while $\mathrm{d} 2$ can be varied in situ by controlling the gas pressure from beneath the substrate film. (c) Extracted phase (orange curves) and group (maroon curves) velocities as a function of $d$. Solid curves are theoretical predictions, while symbols are values determined from the experiment. (d) Quality factor Q (left axis) and propagation length LSPP (right axis) of GPs as a function of d. Symbols are obtained from experimental data, and curves indicate a guide to the eye. The error bars are from different line profiles in one scan image. 

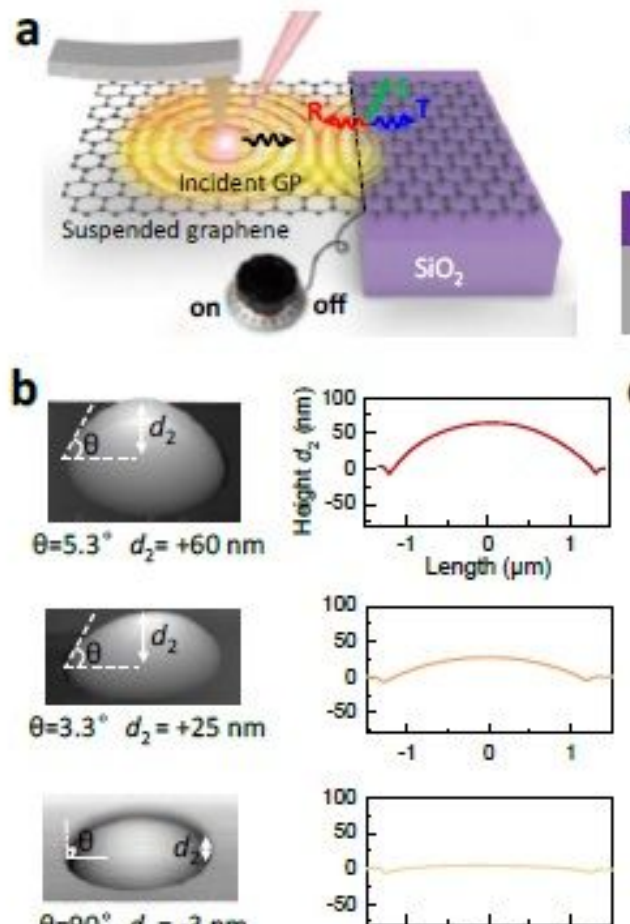

$\theta=90^{\circ} \quad d_{2}=-3 \mathrm{~nm}$
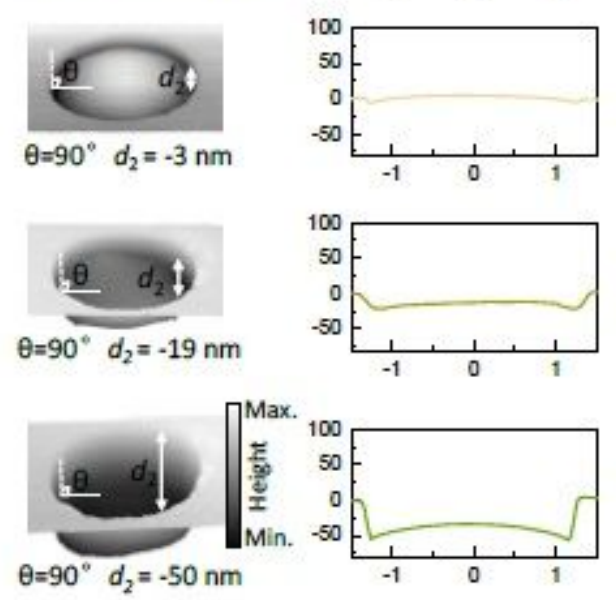
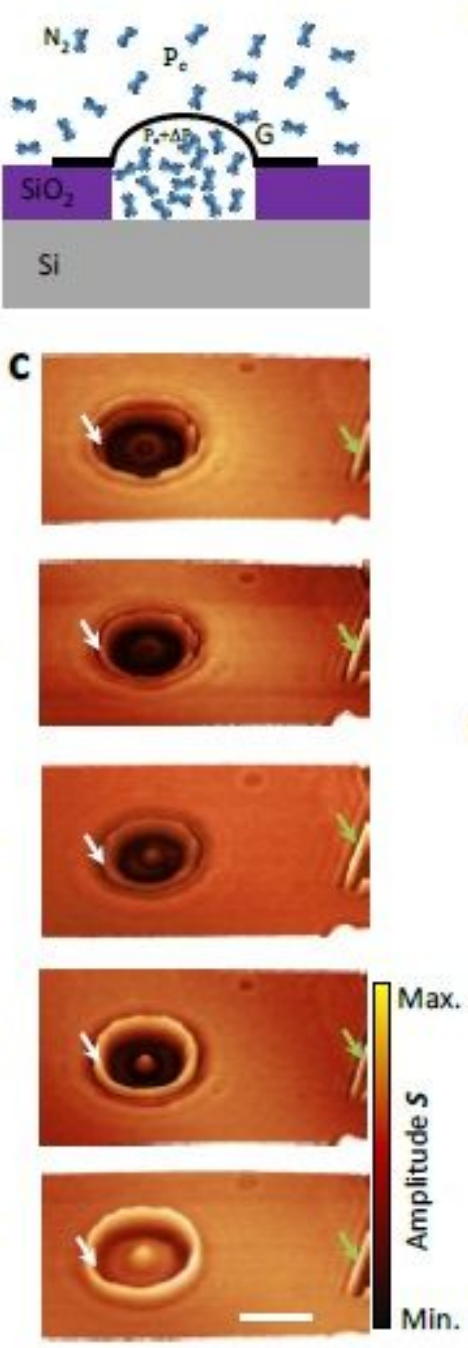

d
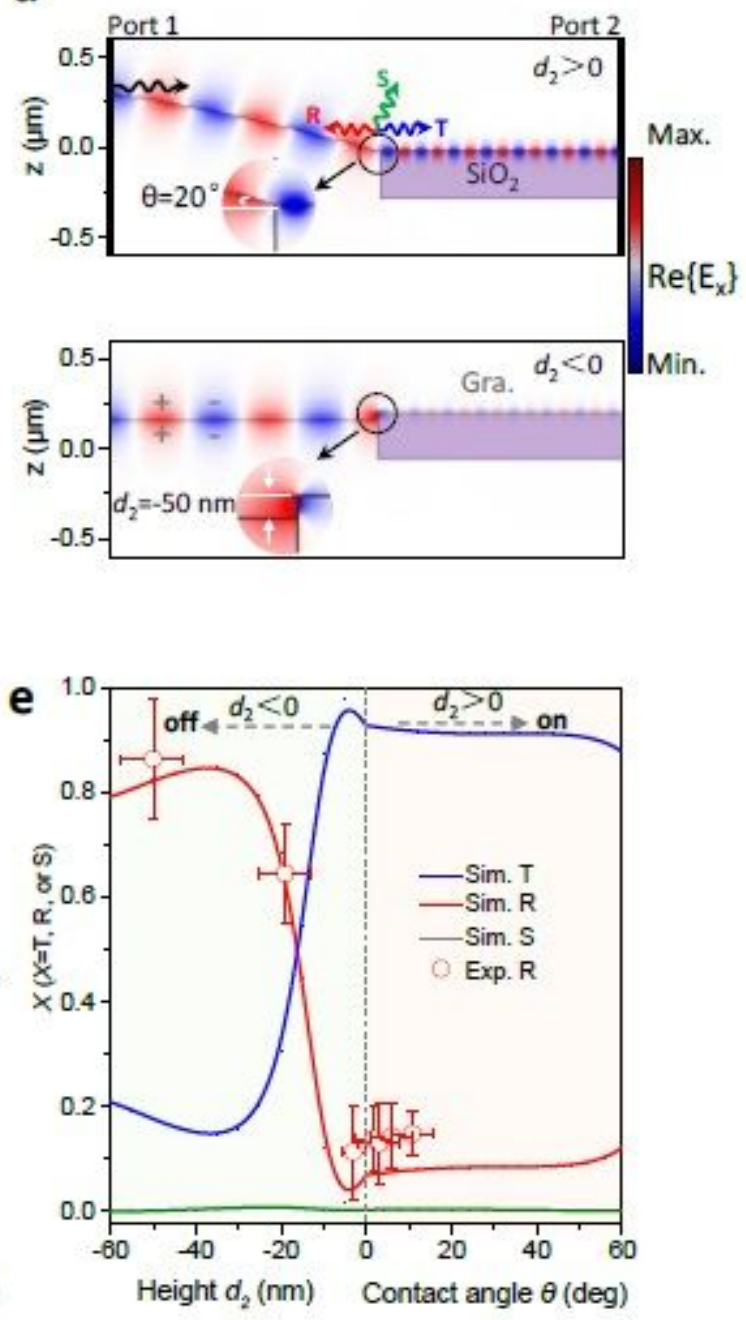

\section{Figure 4}

Plasmonic switch based on tunable control of GP transmission at air-dielectric substrate interfaceinterfaces. (a) Left: a schematic of the plasmonic switch. Black, red, and blue arrows represent incident, reflected, and transmitted plasmons, respectively. The green arrow stands for plasmon scattering (out-coupling) into radiation. Right: schematic of gas diffusion into a graphene bubble from the chamber when applying very high pressure $(\mathrm{Pa})$. Pe indicates the external atmospheric pressure. (b) Left: AFM topography images of suspended graphene with different heights of the graphene bubble d2 obtained by controlling the gas pressure for fixed hole depth $\mathrm{d} 1=300 \mathrm{~nm}$ (see definitions in the inset of Figure $3 b$ ). A negative value of $d 2$ indicates that the graphene bubble is sunken inside the substrate dimple. We use a sample with large $\mathrm{d} 1$ to avoid any complications in the interpretation. Right: Height profiles of suspended graphene bubbles in the left AMF images. (c) Near-field IR images of suspended graphene, taken simultaneously with the AFM topography for the corresponding suspension heights shown in (a). The incident light wavelength is $\lambda 0=10.87 \mu \mathrm{m}(920 \mathrm{~cm}-1)$, and the graphene Fermi energy is $0.40 \pm 0.03 \mathrm{eV}$. Since the entire sample is treated with the same gas concentration and doping time, we assume that the graphene has the same Fermi energy in the substrate and suspended regions. The bright 
fringes at the air-dielectric interface and natural graphene edge are indicated by white and green arrows, respectively. The scale bar indicates $2 \mu \mathrm{m}$. (d) Simulated spatial distribution of the electric field along the $x$-direction as GPs propagate from the suspended region to the region supported by the SiO2 substrate, with d2 $\triangle 0$ (upper part) and d2 $\varangle 0$ (bottom part). Inset: expanded view of the boundary area. (e) Plasmon reflectance (red), transmittance (blue), and scattering (green) as a function of $\mathrm{d} 2$ and $\theta$. Colored curves are numerical simulations with $\mathrm{EF}=0.4 \mathrm{eV}$, whereas symbols represent experimental results extracted from Figure $4 \mathrm{~b}$. Error bars are extracted from different line profiles in each scanned image.

\section{Supplementary Files}

This is a list of supplementary files associated with this preprint. Click to download.

- Editorialpolicychecklist.pdf

- SupplementaryMaterials0422.pdf 